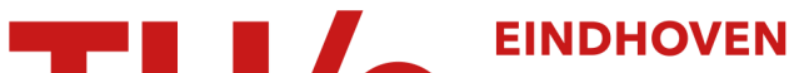 UNIVERSITY OF TECHNOLOGY
}

\section{Ferromagnetic transition in EuS-PbS multilayers}

Citation for published version (APA):

Stachow-Wojcik, A., Story, T., Dobrowolski, W., Arciszewska, M., Galazka, R. R., Kreijveld, M. W., Swüste, C. H. W., Swagten, H. J. M., Jonge, de, W. J. M., Twardowski, A., \& Sipatov, A. Y. (1999). Ferromagnetic transition in EuS-PbS multilayers. Physical Review B, 6O(22), 15220-15229. https://doi.org/10.1103/PhysRevB.60.15220

DOI:

10.1103/PhysRevB.60.15220

Document status and date:

Published: 01/01/1999

\section{Document Version:}

Publisher's PDF, also known as Version of Record (includes final page, issue and volume numbers)

\section{Please check the document version of this publication:}

- A submitted manuscript is the version of the article upon submission and before peer-review. There can be important differences between the submitted version and the official published version of record. People interested in the research are advised to contact the author for the final version of the publication, or visit the $\mathrm{DOI}$ to the publisher's website.

- The final author version and the galley proof are versions of the publication after peer review.

- The final published version features the final layout of the paper including the volume, issue and page numbers.

Link to publication

\section{General rights}

Copyright and moral rights for the publications made accessible in the public portal are retained by the authors and/or other copyright owners and it is a condition of accessing publications that users recognise and abide by the legal requirements associated with these rights.

- Users may download and print one copy of any publication from the public portal for the purpose of private study or research.

- You may not further distribute the material or use it for any profit-making activity or commercial gain

- You may freely distribute the URL identifying the publication in the public portal.

If the publication is distributed under the terms of Article 25fa of the Dutch Copyright Act, indicated by the "Taverne" license above, please follow below link for the End User Agreement:

www.tue.nl/taverne

Take down policy

If you believe that this document breaches copyright please contact us at:

openaccess@tue.nl

providing details and we will investigate your claim. 


\title{
Ferromagnetic transition in EuS-PbS multilayers
}

\author{
A. Stachow-Wójcik \\ Institute of Experimental Physics, Warsaw University, Hoża 69, 00-681 Warsaw, Poland \\ T. Story, W. Dobrowolski, M. Arciszewska, and R. R. Gałązka \\ Institute of Physics, Polish Academy of Sciences, Al. Lotników 32/46, 02-668 Warsaw, Poland \\ M. W. Kreijveld, C. H. W. Swüste, H. J. M. Swagten, and W. J. M. de Jonge \\ Department of Physics and COBRA, Eindhoven University of Technology, 5600MB Eindhoven, The Netherlands \\ A. Twardowski \\ Institute of Experimental Physics, Warsaw University, Hoża 69, 00-681 Warsaw, Poland \\ A. Yu. Sipatov \\ Department of Metal and Semiconductor Physics, Kharkov State Polytechnical University, 21 Frunze Str., 310002 Kharkov, Ukraine
}

(Received 3 March 1999)

\begin{abstract}
The magnetic properties of multilayers of ferromagnetic EuS intercalated with diamagnetic $\mathrm{PbS}$ were studied as a function of the EuS layer thickness (varying from 2 to $200 \mathrm{ML}$ ). The critical temperature $T_{C}$ of the paramagnet-ferromagnet phase transition was determined from magnetization vs temperature measurements and was found to depend on the underlying substrate $\left[\mathrm{KCl}(100) \mathrm{vs}^{\mathrm{BaF}_{2}}(111)\right]$ as well as on the thickness of the EuS layer. For thick layers $\left(d_{\mathrm{EuS}} \approx 200 \mathrm{ML}\right)$, which mimic semibulk EuS, the $T_{C}$ values were found shifted with respect to the bulk EuS (about $1 \mathrm{~K}$ up for layers grown on $\mathrm{KCl}$ and about $3 \mathrm{~K}$ down for layers grown on $\mathrm{BaF}_{2}$ ). This effect is attributed to stress resulting mainly from the difference of thermal expansion coefficients between the substrate and the structure. For thin layers $\left(d_{\text {Eus }}<10 \mathrm{ML}\right)$, a systematic reduction of $T_{C}$ with decreasing EuS layer thickness was observed. This behavior is discussed from two points of view: (a) the reduction of the average number of magnetic neighbors because of the increasing role of the interface for the thin layers, and (b) the three-dimensional/two-dimensional (3D/2D) crossover from a 3D Heisenberg-type ferromagnet to a 2D $X Y$ or Ising-like system. The dependence of the magnetic anisotropy on the EuS layer thickness was studied by ferromagnetic resonance measurements. The energy of magnetic anisotropy can be well described as a sum of a thickness-independent (volume) part and a $1 / d_{\text {EuS }}$ (surface) term. We found that EuS layers with $d_{\text {EuS }}>2 \AA$ magnetize in the plane of the structure. [S0163-1829(99)03045-3]
\end{abstract}

\section{INTRODUCTION}

In recent years a number of studies focused on structures built of magnetic epilayers intercalated with nonmagnetic material (see, e.g., Refs. 1-3). Such systems offer several attractive fundamental challenges, in particular, dimensional effects in magnetism [three-dimensional (3D) to 2D crossover], the oscillatory coupling of magnetic layers via a nonmagnetic medium, the thickness dependence of the magnetic anisotropy of thin layers, giant magnetoresistance effects, or the strain effects resulting from the magnetic/nonmagnetic layers lattice mismatch. ${ }^{1-9}$ So far, most of the investigations were done for magnetic/nonmagnetic structures built of metals, although other systems like oxidic multilayers with nonmetallic spacers (e.g., $\mathrm{Fe}_{3} \mathrm{O}_{4}-\mathrm{MgO}$ ) were studied as well. ${ }^{10}$ Much less is known about the systems built of semiconductors. A particularly interesting possibility is related to the magnetic structures build of europium chalcogenides. These materials, $\mathrm{Eu} X(X=\mathrm{O}, \mathrm{S}, \mathrm{Se}$, and $\mathrm{Te})$, are magnetic semiconductors, which crystallize in the rocksalt structure with the lattice parameter increasing when moving from oxide to telluride. ${ }^{11-16}$ The half-filled $4 f$ level of $\mathrm{Eu}^{2+}$ is located between the valence and the conduction bands ${ }^{17}$ giving rise to a spin-only magnetic moment associated with the $\mathrm{Eu}^{2+}$ ions $(S=7 / 2)$. From magnetic point of view, $\mathrm{Eu} X$ represents the system of localized magnetic moments coupled by exchange interaction well modeled by an isotropic Heisenberg-type Hamiltonian. ${ }^{13,15}$ The character of the magnetic order observed in $\mathrm{Eu} X$ depends crucially on the anion: EuTe is an antiferromagnet, ${ }^{15,18,19}$ EuSe shows both antiferromagnetic and ferromagnetic order depending on the temperature range, ${ }^{15,20}$ and both EuS and EuO are ferromagnets. ${ }^{11,15,21}$ Each $\mathrm{Eu}^{2+}$ ion in the EuS lattice is ferromagnetically coupled to its 12 nearest magnetic neighbors $(\mathrm{NN})\left(J_{\mathrm{NN}} / k_{B}\right.$ $=+0.22 \mathrm{~K})$ and is antiferromagnetically coupled to its six next-nearest neighbors $(\mathrm{NNN})\left(J_{\mathrm{NNN}} / k_{B}=-0.10 \mathrm{~K}\right) .^{15,22,23}$ Interactions beyond $\mathrm{NN}$ and $\mathrm{NNN}$ are believed to be of minor importance. Bulk EuS orders ferromagnetically at $T_{C}$ $=16.6 \mathrm{~K}$.

Recently, there has been an increasing interest in the investigations of thin films of $\mathrm{Eu} X$, in particular with respect to the EuS system that in bulk is considered as an archetype Heisenberg ferromagnet. ${ }^{13,22-26}$ Until now, the properties of antiferromagnetic/nonmagnetic EuTe-PbTe structures have been studied, for which an interlayer coupling, ${ }^{6,27}$ a decrease of Néel temperature with decreasing EuTe layer thickness, ${ }^{28}$ 
and anisotropy effects ${ }^{28-31}$ were found. A ferromagnetic/ nonmagnetic multilayer system of EuS intercalated with insulating SrS has been studied as well. ${ }^{32}$ However, because of the severe interdiffusion due to the very high temperatures used for the deposition of the films (about $900^{\circ} \mathrm{C}$ ), the reported samples turned out to be of insufficient quality to study the effects mentioned above. Recent work on thin EuS layers concerning EuS-Fe and EuS-Cr bilayers yielded interesting results but faced the problem of poor epitaxial compatibility of metals and EuS. ${ }^{25,33}$ In view of this, a most natural nonmagnetic spacer for such structures is the wellknown IV-VI semiconducting compound - lead sulfide $(\mathrm{PbS})$. Lead sulfide crystallizes in the same cubic (rocksalt) crystal structure as EuS, and a lattice mismatch between EuS and $\mathrm{PbS}$ is only $0.5 \%\left(a_{0}^{\mathrm{EuS}}=5.97 \AA\right.$ and $a_{0}^{\mathrm{PbS}}=5.94 \AA$ at $T=300 \mathrm{~K}$ ).

Since $\mathrm{PbS}$ is a typical diamagnet, magnetically the EuS$\mathrm{PbS}$ multilayers form all-semiconducting ferromagnetdiamagnet nanostructures. From the point of view of the electronic structure and the optical properties EuS-PbS multilayers form $\mathrm{PbS}$ multiple quantum well (or superlattice) with the fundamental electronic transitions in the infrared. ${ }^{34-36}$ Since in this structure EuS is a semiconductor with much larger energy gap, EuS is an electron barrier material. EuS crystals usually show semi-insulating electric properties, whereas $\mathrm{PbS}$ is a well-known narrow gap semiconductor with low carrier concentration and semimetallic character of electric conductivity. ${ }^{34}$

The EuS-PbS multilayers are ferromagnetic nanostructures combining both the simple magnetic system (local spin-only magnetic moments in an insulating crystal coupled via short-range exchange interactions), a well-known cubic crystal structure, and a good epitaxial compatibility of both ferromagnetic and diamagnetic layers. Therefore, these structures can be considered as the model low-dimensional nonmetallic Heisenberg ferromagnets. In this paper, we will discuss the dependence of the magnetic properties (the Curie temperature, the magnetic anisotropy) of EuS layers on the thickness of the layer $d_{\text {EuS }}$ and the substrate-induced stress present in the structure. The multilayers studied by us consisted typically of five thin (2-30 ML) layers of EuS separated by a relatively thick nonmagnetic layer of $\mathrm{PbS}$, so each of the layers is magnetically uncoupled to neighboring layers. We will report the results of magnetization, magnetic susceptibility, and ferromagnetic resonance studies of EuS$\mathrm{PbS}$ multilayers in a wide range of EuS layer thicknesses. The outline of the paper is as follows. The samples and the experimental techniques are presented in Sec. II. In Sec. III, the effect of stress on the temperature of ferromagneticparamagnetic phase transition is presented for thick EuS layers $\left(d_{\mathrm{EuS}} \approx 200 \mathrm{ML}\right)$ and is semiquantitatively analyzed. The next two sections (Sec. IV and Sec. V) present the analysis of the dependence of the ferromagnetic transition temperature $T_{C}\left(d_{\text {EuS }}\right)$, and the magnetic anisotropy $K\left(d_{\text {EuS }}\right)$ on the thickness of EuS layer.

\section{EXPERIMENT}

The epitaxial multilayer EuS-PbS structures were grown by thermal evaporation of $\mathrm{PbS}$ from a tungsten boat and by electron-beam evaporation of $\mathrm{EuS}$ in oil-free vacuum $\left(10^{-6}-10^{-7}\right.$ Torr), and their sequential condensation on monocrystalline $\mathrm{KCl}$ (100) or $\mathrm{BaF}_{2}$ (111) substrates at $250{ }^{\circ} \mathrm{C}$. The layer thickness and the growth rate were monitored in situ by a calibrated quartz resonator. The quality of the layers and the superlattice period were checked by x-ray diffraction. Typically, the rocking curves with full width at half maximum of about 300 arcsec and the diffraction patterns with satellite peaks of second and third order were observed. The number of EuS and PbS monolayers in our samples was calculated from the layer thickness divided by the appropriate interlayer distance $\left(a_{0} / 2\right.$ for layers on $\mathrm{KCl}$ substrate; $a_{0} / \sqrt{3}$ for layers on $\mathrm{BaF}_{2}$ substrate). The thickness of EuS and of PbS layers was determined with the accuracy of $1 \mathrm{ML}$. The interdiffusion in such types of EuS-PbS structures was previously investigated by x-ray diffraction profile changes as a function of annealing temperature, yielding an intermixed region of roughly $2 \mathrm{ML} \cdot{ }^{37}$ It was found from transport measurements that our EuS layers are semiinsulating, which indicates a good stoichiometry of the crystals. The EuS-PbS structures grown on $\mathrm{BaF}_{2}$ showed a strong luminescence from $\mathrm{PbS}$ quantum wells. ${ }^{38,39}$ In contrast, no luminescence was observed in the structures grown on $\mathrm{KCl}$. Since the lattice mismatch between the substrate and the multilayer is rather large $\left(\Delta a / a=4 \%\right.$ for $\mathrm{BaF}_{2}$ and $\Delta a / a$ $=6 \%$ for $\mathrm{KCl}$-based structures) the first layer grown on the substrate is a $\mathrm{PbS} 200-700-\AA$-thick buffer layer. It is expected (based on the electron microscopy study of the substrate- $\mathrm{PbS}$ interface) that about 30-50 $\AA$ of $\mathrm{PbS}$ buffer layer contains a lot of structural defects that accommodate the elastic energy due to the lattice mismatch.

For the experiments, the samples of typical area 2 $\times 6 \mathrm{~mm}^{2}$ were cleaved from the center of the 15 $\times 15 \mathrm{~mm}^{2}$ wafers. We studied two series of EuS-PbS multilayer samples with a similar spectrum of layer thickness, but differing by layer orientation and the type of the substrate $\left[\mathrm{KCl}(100)\right.$ or $\mathrm{BaF}_{2}$ (111)]. The parameters of all the investigated structures are collected in Table I. The EuS layer thickness was ranging from 2 to $24 \mathrm{ML}$, while the $\mathrm{PbS}$ layers were kept relatively thick and nearly constant $\left(d_{\mathrm{PbS}}\right.$ $\approx 50 \mathrm{ML}$ ). In each multilayer the EuS-PbS bilayer was repeated 5 or 10 times. Additionally, a few thick EuS-PbS bilayers $\left(d_{\mathrm{EuS}} \approx 200 \mathrm{ML}\right)$ were grown on $\mathrm{KCl}(100)$ and $\mathrm{BaF}_{2}(111)$ as a reference to bulk EuS (Table I).

The Cryogenic superconducting quantum interference device (SQUID) magnetometer with the sensitivity of $10^{-7}$ emu was used to measure magnetization as a function of temperature, $M(T)$, in the temperature range from 2 to 30 $\mathrm{K}$. Providing magnetic fields up to $B=6 \mathrm{~T}$ this device was unable to arrive at the true zero magnetic field in the superconducting coil. To take the residual field under control, measurements were performed at the fixed field of $B$ $=1 \mathrm{mT}$. The Lake Shore ac magnetometer was used to study the temperature dependence of ac magnetic susceptibility, $\chi(T)$. The measurements were performed within the temperature range $7-22 \mathrm{~K}$, at $B=10 \mu \mathrm{T}$, alternating with the frequency $f=995 \mathrm{~Hz}$. In all the above mentioned experiments, the magnetic field was applied in the plane of EuS-PbS structure and correction for demagnetization field was not made.

Apart from the magnetization and the magnetic suscepti- 
TABLE I. The EuS-PbS multilayers studied in this work. (For the layers with $n>10$ ML the thickness is rounded to the nearest integer.)

\begin{tabular}{lccc}
\hline \hline Thickness (in monolayers) & $\begin{array}{c}\text { Number of } \\
\text { EuS-PbS } \\
\text { periods }\end{array}$ & $\begin{array}{c}\text { Substrate } \\
\text { and orientation }\end{array}$ \\
\hline$d_{\text {EuS }}$ & $d_{\mathrm{PbS}}$ & 5 & $\mathrm{KCl}(100)$ \\
2.0 & 59 & 5 & $\mathrm{KCl}(100)$ \\
5.7 & 57 & 5 & $\mathrm{KCl}(100)$ \\
6.7 & 59 & 5 & $\mathrm{KCl}(100)$ \\
10 & 57 & 5 & $\mathrm{KCl}(100)$ \\
18 & 52 & 5 & $\mathrm{KCl}(100)$ \\
22 & 59 & 10 & $\mathrm{KCl}^{2}(100)$ \\
167 & 52 & 1 & $\mathrm{KCl}^{2}(100)$ \\
201 & 270 & 1 & $\mathrm{KCl}^{2}(100)$ \\
& 152 & 5 & \\
1.7 & & $\mathrm{BaF}_{2}(111)$ \\
2.3 & 51 & 5 & $\mathrm{BaF}_{2}(111)$ \\
2.9 & 50 & 5 & $\mathrm{BaF}_{2}(111)$ \\
4.4 & 45 & 5 & $\mathrm{BaF}_{2}(111)$ \\
5.8 & 51 & 5 & $\mathrm{BaF}_{2}(111)$ \\
8.7 & 50 & 5 & $\mathrm{BaF}_{2}(111)$ \\
16 & 45 & 5 & $\mathrm{BaF}_{2}(111)$ \\
23 & 51 & 5 & $\mathrm{BaF}_{2}(111)$ \\
203 & 50 & 1 & $\mathrm{BaF}_{2}(111)$ \\
\hline \hline
\end{tabular}

bility measurements, the ferromagnetic resonance (FMR) was studied using a Bruker $X$-band spectrometer $(f$ $=9.45 \mathrm{GHz}$ ) equipped with an Oxford Instruments lowtemperature continuous flow cryostat. Since this device covers the temperature range $4 \leqslant T \leqslant 300 \mathrm{~K}$ and magnetic field range $B \leqslant 2 \mathrm{~T}$, it excellently fits the requirements for FMR measurements of EuS layers $\left(T_{C}^{\mathrm{bulk}}=16.6 \mathrm{~K}\right.$, maximum demagnetization field about $1.5 \mathrm{~T}$ ).

To study the ferromagnetic phase transition, the temperature dependence of magnetization $M(T)$ was measured at low field $(B=1 \mathrm{mT})$. The critical temperature $T_{C}$ was determined as the temperature corresponding to the inflection point of the low-field $M(T)$ dependence (arrows in Fig. 1). The application of different methods for determining the $\mathrm{Cu}$ rie temperature [Arrot plots, phenomenological $M(T) \propto\left(T_{C}\right.$ $-T)^{1 / 2}$ dependence] yield about the same result with the accuracy of a few tenths of Kelvin, which is almost an order of magnitude smaller than the $T_{C}$ changes we present in this work.

All our experimental data (the temperature and magnetic field dependence of magnetization, the temperature dependence of magnetic susceptibility, and the temperature and the angle dependence of ferromagnetic resonance) clearly indicate a ferromagnetic transition even for multilayers with EuS thickness of only $2 \mathrm{ML}$. The thickness of the EuS layer is, however, one of the primary factors determining the actual ferromagnetic transition temperature as will be discussed below.

\section{THE EFFECT OF STRESS}

To provide a reference for our multilayers, we have investigated thick EuS-PbS epilayers $\left(d_{\mathrm{EuS}} \approx 200 \mathrm{ML}\right.$, see

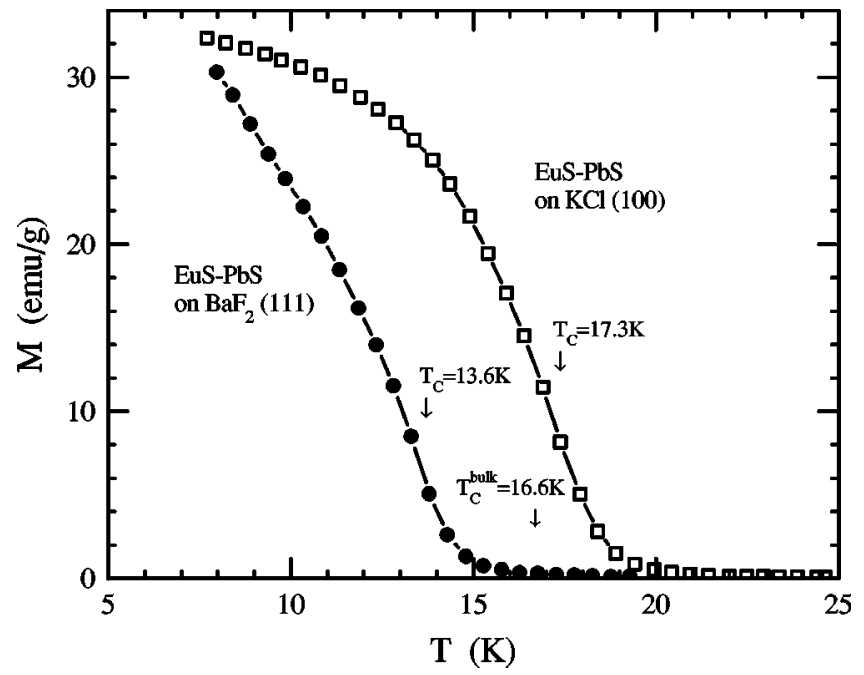

FIG. 1. Temperature dependence of low-field magnetization $(B=1 \mathrm{mT})$ of $\operatorname{EuS}(201 \mathrm{ML})-\mathrm{PbS}(152 \mathrm{ML})$ bilayer grown on the $\mathrm{KCl}$ (100) substrate (open squares) and for the $\operatorname{EuS}(203 \mathrm{ML})$ $\mathrm{PbS}\left(161 \mathrm{ML}\right.$ ) bilayer grown on the $\mathrm{BaF}_{2}$ (111) substrate (full circles). The solid lines are guides to the eye only. The ferromagnetic temperatures $T_{C}$ were determined as the inflection point of the $M(T)$ curve. The $T_{C}^{\text {bulk }}=16.6 \mathrm{~K}$ for bulk EuS is also shown.

Table I) grown both on $\mathrm{BaF}_{2}$ and $\mathrm{KCl}$ substrates. The ferromagnetic transition temperatures $T_{C}$ were found to be shifted with respect to the critical temperature of the bulk EuS crystal $T_{C}^{\text {bulk }}=16.6 \mathrm{~K}$. Moreover, the temperature shift $\Delta T_{C}$ $=T_{C}-T_{C}^{\text {bulk }}$ appeared to be substrate dependent. For thick EuS-PbS bilayers grown on the $\mathrm{KCl}(100)$ substrate $\Delta T_{C}$ is about $+1 \mathrm{~K}$ (i.e., increase of critical temperature). In contrast, for the EuS-PbS bilayer with similar thicknesses, but grown on $\mathrm{BaF}_{2}$ (111), $\Delta T_{C}$ is about $-3 \mathrm{~K}$ (i.e., decrease of critical temperature). In Fig. 1 the results for two samples on two different substrates are presented. We attribute the observed shift of critical temperature $\Delta T_{C}$ to the in-plane stress present in our EuS-PbS structures. This stress changes the Eu-Eu distances and this in turn influences the spin-spin exchange integrals $J_{i}$, eventually changing the ferromagnetic transition temperature $T_{C}\left(T_{C} \propto \sum z_{i} J_{i}\right.$, where $i$ corresponds to the number of coordinate sphere and $z_{i}$ is the number of magnetic neighbors ${ }^{40}$ ). One can expect that the opposite sign of $\Delta T_{C}$ for EuS-PbS layers grown either on $\mathrm{KCl}$ (100) or on $\mathrm{BaF}_{2}$ (111) corresponds to a different kind of lattice deformation.

The main sources of stress that have to be taken into account in the case of our heterostructures are the substratemultilayer lattice mismatch, the EuS-PbS lattice mismatch, and the difference between the thermal expansion coefficients (TEC) of the substrate and the multilayer. At the growth temperature $\left(T_{g}=520 \mathrm{~K}\right)$, the stress due to the lattice mismatch $(\Delta a / a)$ between the $\mathrm{KCl}$ and $\mathrm{PbS}$ buffer layer or between the $\mathrm{BaF}_{2}$ and $\mathrm{PbS}$ buffer is expected to be relaxed by the creation of structural defects (3D island-type growth) in a thin $\mathrm{PbS}$ layer at the interface of the substrate/PbS buffer. It is known that even for quite thick buffer layers, in many semiconductor strained heterostructures, the relaxation of the substrate-buffer mismatch-induced strain might be not complete. ${ }^{41}$ However, since the lattice constants of both $\mathrm{KCl}$ $(6.29 \AA)$ and $\mathrm{BaF}_{2}(6.20 \AA)$ are larger than the lattice con- 
stants of PbS (5.94 $\AA$ ) and EuS $(5.97 \AA)$, the action of this source of stress would result in qualitatively the same behavior (i.e., lowering of the transition temperature) for structures grown on either substrate. Therefore, this effect cannot account for our experimental findings. Due to the small lattice mismatch between EuS and $\mathrm{PbS}(0.5 \%)$, these layers grow pseudomorphically on top of each other up to more than $1000 \AA$, and the strain in these structures is shared (in the ideal situation) by EuS and $\mathrm{PbS}$ layers in accordance with their lattice constants, thicknesses, and elastic constants. ${ }^{41}$ However, it is well known from the low-temperature optical and transport studies of related $\mathrm{PbTe} / \mathrm{BaF}_{2}$ and $\mathrm{PbSe} / \mathrm{BaF}_{2}$ IV-VI heterostructures, that in these layers the more important source of stress is the difference between the TEC of the substrate and the layer. ${ }^{42}$ Since the substrate, the PbS buffer, and the rest of the multilayer are elastically strongly coupled layers, the lowering of the temperature from the growth temperature $\left(T_{g}=520 \mathrm{~K}\right)$ down to the temperature range of ferromagnetic phase transition $\left(T_{C}=10-20 \mathrm{~K}\right)$ might result in a lattice mismatch due to the different temperature dependence of TEC of the substrate and the multilayer.

The effect of TEC stress was calculated for the temperature interval from $T_{g}=520 \mathrm{~K}$ down to $T_{C}=10 \mathrm{~K}$ for EuS$\mathrm{PbS}$ bilayers grown on $\mathrm{KCl}$ and on $\mathrm{BaF}_{2}$. In our calculations, we adopted the simple model ${ }^{41}$ assuming that (1) at the growth temperature $T_{g}=520 \mathrm{~K}$ the stress due to the lattice mismatch between substrate and $\mathrm{PbS}$ buffer is fully relaxed, and (2) all the TEC stress builds in the structure when the sample is cooled down (no possibility for the formation of new defects) ${ }^{41}$ The neglegible role of the relaxation of TEC stress by the formation of defects is confirmed by the lack of the dependence of the magnetic properties of EuS-PbS on thermal cycling between room temperature and liquid helium temperature.

For the case of the EuS-PbS structure on the $\mathrm{KCl}$ (100) substrate, we expect the following thermal history. The TEC of $\mathrm{KCl}\left(\alpha_{\mathrm{KCl}}=37 \times 10^{-6} \mathrm{~K}^{-1}\right.$ at $\left.T=300 \mathrm{~K}\right)$ is substantially larger than the TEC of $\mathrm{PbS}\left(\alpha_{\mathrm{PbS}}=20 \times 10^{-6} \mathrm{~K}^{-1}\right.$ at $T=300 \mathrm{~K}$ ). This means that with lowering the temperature the $\mathrm{KCl}$-substrate shrinks faster than the PbS-buffer layer and the EuS-PbS structure is subjected to compressing (in plane) biaxial stress increasing with decreasing temperature. In our calculations we took into account the temperature dependence of TEC of the substrate and the TEC of the PbS buffer layer. This mismatch of TEC's causes the difference $\left(\Delta a_{\mathrm{TEC}}\right)$ between the reduction of lattice constant of $\mathrm{KCl}$ $\left(\Delta a_{\mathrm{KCl}}\right)$ and PbS-buffer $\left(\Delta a_{\mathrm{PbS}}\right)$, when the temperature lowers from $T_{g}=520 \mathrm{~K}$ to $T=10 \mathrm{~K}$. Because the substrate is much thicker $(\approx 1 \mathrm{~mm})$ than the buffer $(<0.1 \mu \mathrm{m})$, we assume that practically all the TEC stress will accumulate in $\mathrm{PbS}$-buffer layer. In this (KCl-substrate) case of EuS-PbS structure, the bulk lattice constant of $\mathrm{PbS}$, at low temperatures, will be then reduced by the value of $\Delta a_{\mathrm{TEC}}=\left(\Delta a_{\mathrm{KCl}}\right.$ $\left.-\Delta a_{\mathrm{PbS}}\right)<0$. This "new" in-plane lattice constant $a_{\|}$will be set for both $\mathrm{PbS}$ and EuS layers $a_{\|}=a_{\|}^{\mathrm{PbS}}=a_{\|}^{\mathrm{EuS}}$. Our estimation yields the in-plane lattice constant of EuS layer $a_{\|}^{\text {EuS }}$, which is about $1 \%$ shorter as compared to the bulk material $a_{0}^{\mathrm{EuS}}$ at $T=10 \mathrm{~K}$. The accompanying deformation of the EuS unit cell along the normal to the plane of the layer (Poisson effect) was determined by using the common stressstrain relations in cubic crystals, with boundary condition of zero stress perpendicular to the plane (100) of EuS layer and biaxial stress in the plane of the layer. ${ }^{41}$ Using this calculation, the ratio of the in-plane strain to the perpendicular one $e_{\perp} / e_{\|}=-0.17$ was found.

In the molecular field approach, the ferromagnetic critical temperature $T_{C}$ for EuS is related to the exchange integrals $J_{\mathrm{NN}}$ (nearest neighbors) and $J_{\mathrm{NNN}}$ (next-nearest neighbors) through the following expression: $k_{B} T_{C}=\alpha\left(12 J_{\mathrm{NN}}\right.$ $\left.+6 J_{\mathrm{NNN}}\right)$, where $\alpha=2 S(S+1) / 3=10.5$ for Eu ions. In our calculations, the change of $T_{C}$ results from the changes of the interspin distances between $12 \mathrm{NN}$ magnetic ions. The effect due to $6 \mathrm{NNN}$ is not important since the experimental data suggest that in Eu chalcogenides the $J_{\mathrm{NNN}}$ exchange integral is only weakly dependent on lattice constant, ${ }^{40}$ $d J_{\mathrm{NNN}}(a) / d a \approx 0$. In that case, the change of $T_{C}$ is given by $\Delta T_{C}(a) \approx \alpha\left[d J_{\mathrm{NN}}(a) / d a\right] \Delta a_{\mathrm{eff}}$, where $\Delta a_{\mathrm{eff}}=\left[4 \Delta a_{\|}\right.$ $\left.+8\left(\Delta a_{\perp} / 2+\Delta a_{\|} / 2\right)\right]$ and $\Delta a_{\text {eff }} / \sqrt{2}$ is the effective change of distances of all $12 \mathrm{NN}$ to given Eu ion [4 NN in the (100) plane and $8 \mathrm{NN}$ in neighboring planes], affected by the inplane biaxial stress. The obtained result is $\Delta a_{\text {eff }}$ $=7.32 \Delta a_{0}^{\mathrm{EuS}}$, where $\Delta a_{0}^{\mathrm{EuS}}$ is the difference between the inplane strained lattice constant $a_{\|}^{\text {EuS }}$ and $a_{0}^{\text {EuS }}$ of the unstrained bulk EuS (compare with the case of homogenous hydrostatic pressure, when $\Delta a_{\mathrm{eff}}=12 \Delta a_{0}^{\mathrm{EuS}}$ ). The reduction is due to the opposite changes of the perpendicular and in-plane lattice parameters $\left(e_{\perp} / e_{\|}<0\right)$, which influences the interspin distances between NN located in different lattice planes. The lattice constant dependence of the $\mathrm{NN}$ exchange parameter $d J_{\mathrm{NN}}(a) / d a$ was taken [assuming $d J_{\mathrm{NNN}}(a) / d a \approx 0$ ] from the high-pressure neutron diffraction measurements of a bulk EuS. ${ }^{40}$ In the range of interatomic distances corresponding to our case, the changes of $T_{C}$ were found to be $d T_{C}(a) / d a$ $=-(133 \pm 7) \mathrm{K} / \AA$. Using the neutron scattering data on $d T_{C} / d a$ as well as the calculated $\Delta a_{\text {eff }}$ induced by TEC, we are now able to estimate the shift $\Delta T_{C}$ of the critical temperature of EuS-PbS structure on $\mathrm{KCl}$ (100) substrate with respect to $T_{C}$ of bulk EuS, yielding $\Delta T_{C}=+6.2 \mathrm{~K}$.

It has to be noticed that our estimation provides the upper limit for the considered effect since we assumed that the complete (no partial relaxation) TEC-induced stress is present in the structure. The calculated increase of $T_{C}$ is larger than the one obtained in the experiment (Fig. 1). It suggests that the stress might be partially relaxed, e.g., in the form of lattice defects, during the cooling process from the growth to room temperature and further down to the ferromagnetic region. Preliminary results of x-ray diffraction studies of the lattice constant $\left(a_{\perp}\right)$ perpendicular to the (100) plane of EuS-PbS/KCl multilayer suggest that our structures are stressed at $T=300 \mathrm{~K}$ in a way expected for the action of the TEC-induced stress. To our knowledge, there are no lowtemperature $\mathrm{x}$-ray diffraction data available for the in-plane lattice parameter that would provide a direct quantitative measure of the lattice deformation.

Similarly as above, one can calculate $\Delta T_{C}^{f}$ for the freestanding pseudomorphic EuS-PbS multilayer (without $\mathrm{KCl}$ substrate) when the only source of stress is the difference between the lattice constants of bulk crystals of EuS and $\mathrm{PbS}$ (at low temperatures). For layers with similar elastic constants and equal thickness $\left(d_{\mathrm{EuS}} \approx d_{\mathrm{PbS}}\right)$, roughly half of the lattice mismatch stress builds in each of them. ${ }^{28,41}$ The result 

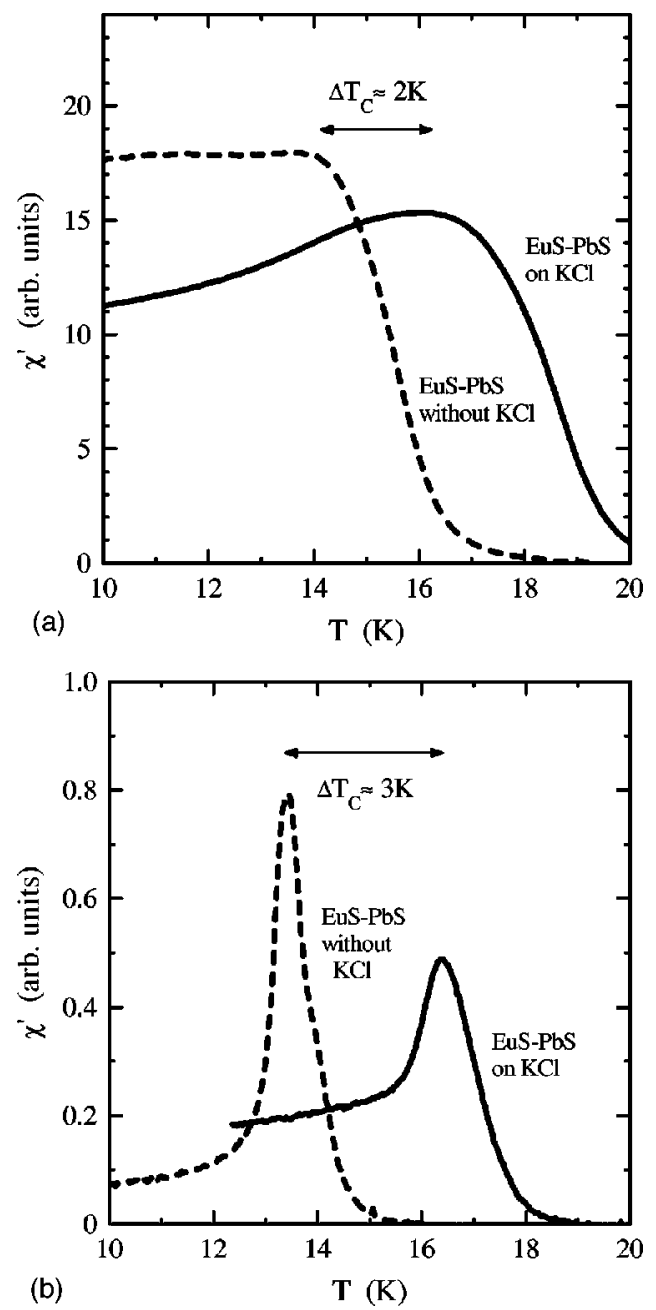

FIG. 2. Temperature dependence of ac magnetic susceptibility for the $\operatorname{EuS}(167 \mathrm{ML})-\mathrm{PbS}(270 \mathrm{ML})$ bilayer (a) and the $[\mathrm{EuS}(18$ ML)-PbS(59 ML)] multilayer (b). The solid lines show the data for layers on the $\mathrm{KCl}(100)$ substrates, whereas the dashed lines show the data for the same layers without substrate.

for the free-standing EuS-PbS structure grown in the [100] direction is $\Delta T_{C}^{f}=+1.7 \mathrm{~K}$. This indicates that $T_{C}$ of strained EuS-PbS/KCl could be shifted at most with $+4.5 \mathrm{~K}$ $\left(\Delta T_{C}-\Delta T_{C}^{f}\right)$ higher with respect to the $T_{C}$ of the pseudomorphically strained free EuS-PbS structure. This result of our calculations was directly verified experimentally. The temperature dependence of the ac magnetic susceptibility $[\chi(T)]$ of EuS-PbS structure on the $\mathrm{KCl}$ substrate was compared with the $\chi(T)$ dependence observed for the same sample without the substrate (free-standing EuS-PbS structure with $\mathrm{KCl}$ substrate removed by dissolving in water). During the measurement, the free EuS-PbS structure holds on the glass substrate by weak van der Waals forces with negligible substrate-layer stress. As shown in Fig. 2, the removal of the $\mathrm{KCl}$ substrate leads to the decrease of the ferromagnetic transition temperature $T_{C}$. Both curves are shifted one from the other by $2-3 \mathrm{~K}$ in a way expected for the action of the stress due to the difference in thermal expansion coefficient of the structure and the substrate.

The case of EuS-PbS on the $\mathrm{BaF}_{2}$ (111) substrate is somewhat different. The TEC of $\mathrm{BaF}_{2}\left(\alpha_{\mathrm{BaF}_{2}}=17 \times 10^{-6} \mathrm{~K}^{-1}\right.$ at $T=300 \mathrm{~K})$ is slightly smaller than that of $\mathrm{PbS}\left(\alpha_{\mathrm{PbS}}\right.$
$=20 \times 10^{-6} \mathrm{~K}^{-1}$ at $\left.T=300 \mathrm{~K}\right)$. This means that the tensile in-plane stress is acting on the PbS-buffer layer with decreasing temperature. The calculations of the in-plane (111) Eu-Eu distance $b$ were carried out in a way analogous to the $\mathrm{KCl}$ case considered above. It turns out that, despite the tensile in-plane stress of the PbS-buffer, the unit cell of the EuS layer is still under in-plane compression due to the EuS-PbS lattice mismatch ( $a_{\|}^{\text {EuS }}$ is $0.3 \%$ shorter than $a_{0}^{\text {EuS }}$ of bulk EuS). The deformation of the EuS unit cell within the (111) plane was found to be $\Delta b_{\text {eff }}=5 \Delta b_{0}^{\text {EuS }}$. It has to be noticed that the relation between the in-plane and perpendicular strain is now given by $e_{\perp} / e_{\|}=-0.75 .{ }^{38} \mathrm{With}$ respect to the bulk EuS the calculated shift of $T_{C}$ for the EuS-PbS structure on $\mathrm{BaF}_{2}$ is $\Delta T_{C}=+0.7 \mathrm{~K}$, while for the free-standing (111) EuS-PbS structure $\Delta T_{C}^{f}=+0.8 \mathrm{~K}$.

Our analysis of the effect of stress on the magnetic properties of EuS-PbS/KCl structures indicates the important role of this effect and shows that the increase of critical temperature observed for layers as compared to the bulk crystals may be well understood. One can demonstrate this effect experimentally in a very straightforward way. The analysis performed for the case of EuS-PbS/ $\mathrm{BaF}_{2}$ structures indicates a much less significant influence of stress-induced changes of interspin distances on critical temperature. Therefore, the effect of thermal stress does not explain the experimentally observed reduction of Curie temperatures of EuS-PbS layers on $\mathrm{BaF}_{2}$ substrates. Since both $\mathrm{KCl}-$ and $\mathrm{BaF}_{2}$-based structures were grown in a single growth procedure, we can disregard the possible chemical changes in the EuS layers (nonstoichiometry, etc.). In our opinion, the factor that may be important for $\mathrm{EuS}-\mathrm{PbS} / \mathrm{BaF}_{2}$ layers is the stress-induced lowering of the crystal lattice symmetry. We expect tetragonal lattice distortion for $\mathrm{KCl}$ (100)-based structures but a lower symmetry (trigonal) distortion for $\mathrm{BaF}_{2}$ (111)-based structures. This difference is the consequence of the "good compatibility" of biaxial symmetry of stress and four-fold symmetry of the (100) plane of rocksalt lattice and the "noncompatible" character of biaxial stress and sixfold symmetry of the (111) plane. The quantification of this suggestion via theoretical calculations of the exchange interactions in EuS in distorted lattice is, to our knowledge, not known.

\section{THICKNESS DEPENDENCE OF THE FERROMAGNETIC TRANSITION TEMPERATURE}

The magnetic properties of two series of EuS-PbS multilayers on $\mathrm{KCl}(100)$ or $\mathrm{BaF}_{2}$ (111) were studied as a function of the thickness of ferromagnetic EuS layer $d_{\text {EuS }}$ varying from 2 to $24 \mathrm{ML}$. Since the PbS layers were relatively thick $\left(d_{\mathrm{PbS}} \approx 50 \mathrm{ML}\right)$, our system can be considered as consisting of noncoupled thin magnetic layers. The thickness of the $\mathrm{PbS}$ layer was practically the same for all the studied structures (see Table I).

The temperature dependence of the low-field $(B$ $=1 \mathrm{mT})$ magnetization $M(T)$ clearly shows a paramagnetic-ferromagnetic phase transition. The representative result for three structures grown on $\mathrm{BaF}_{2}$ substrate is displayed in Fig. 3. The ferromagnetic transition temperature $T_{C}$ clearly depends on the thickness of the magnetic EuS layer. In Fig. 4 we show the $T_{C}\left(d_{\text {EuS }}\right)$ dependence for both series of the investigated EuS-PbS structures. The data for 


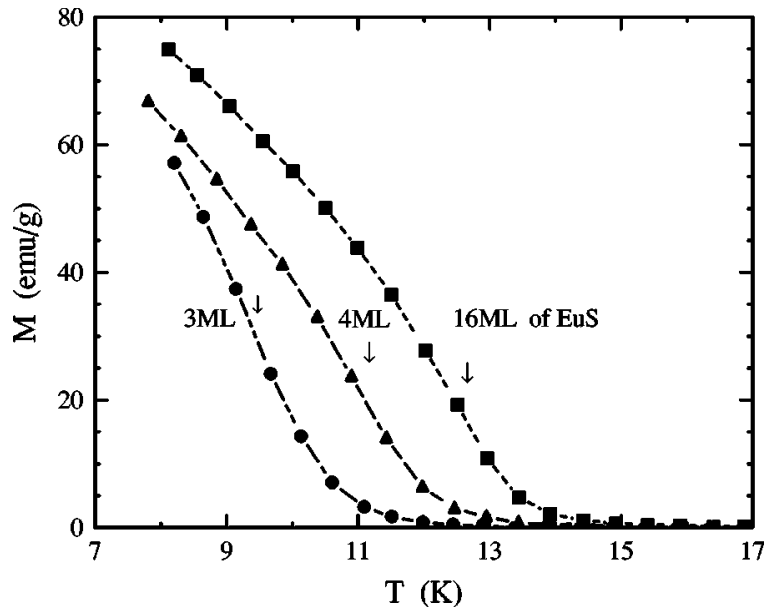

FIG. 3. Temperature dependence of the magnetization measured at low magnetic field $(B=1 \mathrm{mT})$ for three $\mathrm{EuS}-\mathrm{PbS}$ multilayers grown on $\mathrm{BaF}_{2}$ substrate: $\mathrm{EuS}(3 \mathrm{ML})-\mathrm{PbS}(50 \mathrm{ML})$, circles; $\mathrm{EuS}(4$ $\mathrm{ML})-\mathrm{PbS}(51 \mathrm{ML})$, triangles; $\operatorname{EuS}(16 \mathrm{ML})-\mathrm{PbS}(51 \mathrm{ML})$, squares. The dashed lines are guides to eye only. The arrows indicate the ferromagnetic phase transition temperature $T_{C}$ determined as the inflection point of the $M(T)$ curve.

thick $\operatorname{EuS}(\approx 200 \mathrm{ML})-\mathrm{PbS}(\approx 150 \mathrm{ML})$ bilayers are presented as well (see Table I). For both series of structures, the $T_{C}\left(d_{\text {EuS }}\right)$ dependence reveals qualitatively the same behavior. However, the transition temperatures of the EuS-PbS structures on $\mathrm{BaF}_{2}$ are shifted to lower temperatures by about $4 \mathrm{~K}$ with respect to the $\mathrm{KCl}$-based structures. This effect is attributed to the stress present in the EuS-PbS system as discussed in the previous section. For structures with an EuS layer thicker than roughly $10 \mathrm{ML}$, the value of $T_{C}$ is nearly the same (within the accuracy of $T_{C}$ determination, which is $\pm 1 / 4 \mathrm{~K}$ ), and corresponds to the $T_{C}$ of semibulk EuS (thick bilayers) on the $\mathrm{BaF}_{2}$ or on the $\mathrm{KCl}$ substrate. For the struc-

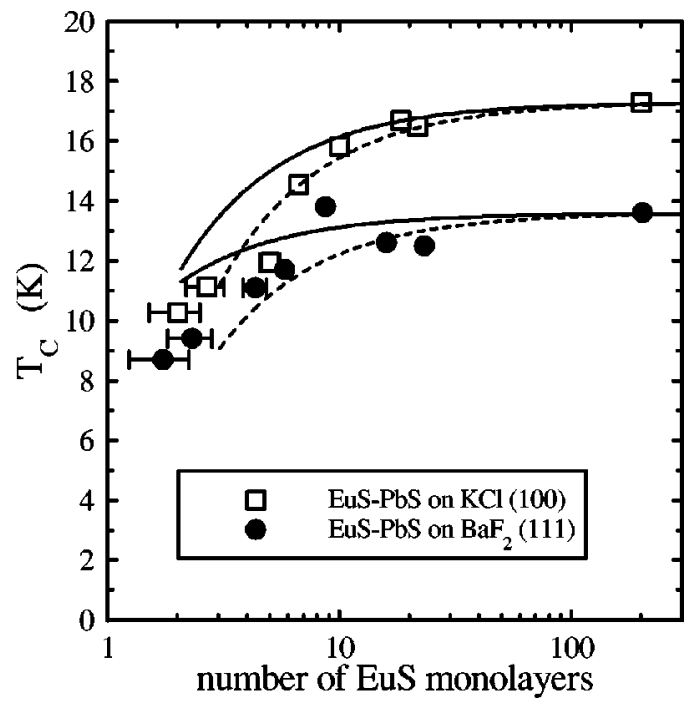

FIG. 4. The thickness dependence of the Curie temperature $T_{C}$ for two series of EuS-PbS multilayers grown on $\mathrm{KCl}(100)$ substrate (open squares), and on $\mathrm{BaF}_{2}$ (111) substrate (full circles). Solid lines correspond to the calculations [Eq. (2)] for the case of sharp EuS-PbS interface, whereas the dashed lines correspond to the case of intermixed interface of 2-ML width.
TABLE II. Parameters used for the calculations of the thickness dependence of the ferromagnetic phase transition temperature $T_{C}^{(n)}$.

\begin{tabular}{lccc}
\hline \hline Substrate & Interface & $T_{C}^{\text {bulk }}$ & Parameter $c$ of Eq. (2) \\
\hline $\mathrm{KCl}$ & sharp & $17.3 \mathrm{~K}$ & $2 / 3$ \\
$\mathrm{KCl}$ & intermixing of $2 \mathrm{ML}$ & $17.3 \mathrm{~K}$ & 1.07 \\
$\mathrm{BaF}_{2}$ & sharp & $13.6 \mathrm{~K}$ & 0.35 \\
$\mathrm{BaF}_{2}$ & intermixing of $2 \mathrm{ML}$ & $13.6 \mathrm{~K}$ & 1.01 \\
\hline \hline
\end{tabular}

tures with EuS layers thinner than $10 \mathrm{ML}, T_{C}$ decreases with decreasing EuS layer thickness (Fig. 4). We attribute this effect primarily to the reduction of an average number of magnetic neighbors for magnetic ions located at the interfaces. Even for the sharp EuS-PbS interface, spins in the outermost monolayers have less magnetic neighbors than spins in the volume of EuS layer. For the realistic case of EuS-PbS interfaces with finite widths, the intermixing of $\mathrm{EuS}$ with $\mathrm{PbS}$ reduces the average number of magnetic neighbors even further. This effect was directly observed in, e.g., NMR studies of the $\mathrm{Co}-\mathrm{Cu}$ interface. ${ }^{44}$

We considered this effect using a so-called "bond-loss" model $^{28,42,43}$ that originates from the mean-field approach. In this model, $k_{B} T_{C}$ is simply proportional to the total groundstate energy of the ferromagnetic state: $k_{B} T_{C} \approx \sum z_{i} J_{i} S^{2}$. In this approximation, the phase transition temperature $T_{C}^{(n)}$ for the layer containing $n$ EuS monolayers scales by the average number of magnetic neighbors $\bar{z}_{i}$ :

$$
T_{C}^{(n)}=T_{C}^{\text {bulk }}\left(\bar{z}_{1} J_{\mathrm{NN}}+\bar{z}_{2} J_{\mathrm{NNN}}\right) /\left(12 J_{\mathrm{NN}}+6 J_{\mathrm{NNN}}\right),
$$

where $\bar{z}_{1}$ and $\bar{z}_{2}$ are the average numbers of nearest neighbors (coupled by $J_{\mathrm{NN}}$ ) and next-nearest neighbors (coupled by $J_{\mathrm{NNN}}$ ), respectively, which depend on the number $n$ of $\mathrm{EuS}$ monolayers in the layer.

The $T_{C}\left(d_{\text {EuS }}\right)$ dependence was analyzed separately for $\mathrm{KCl}$ - and $\mathrm{BaF}_{2}$-based series of EuS-PbS multilayers. The critical temperature of appropriate semibulk (stressed) EuS, which was determined experimentally for the samples with thick EuS layers, was adopted as $T_{C}^{\text {bulk }}$ (Table II). The number of magnetic neighbors $\bar{z}_{1}$ (and $\bar{z}_{2}$ ) was calculated for Eu ions located in each of $n$ monolayers of the EuS layer. The average number of magnetic neighbors, $\bar{z}_{1}(n)$ and $\bar{z}_{2}(n)$, depends on the thickness of the EuS layer, on the growth direction, and on the intermixing profile at the interface (Fig. 5). For example, the Eu ion in the outermost layer at a sharp EuS-PbS interface for $\mathrm{KCl}$ (100)-based structure has only 8 NN and 5 NNN instead of 12 NN and 6 NNN as in bulk EuS, while it has $6 \mathrm{NN}$ and $3 \mathrm{NNN}$ at the (111) sharp EuS-PbS interface for $\mathrm{BaF}_{2}$ (111)-based structure. For the intermixed interface, the average number of magnetic neighbors was calculated assuming a random distribution of Eu ions over the sites of the cation sublattice. The probability of a given site to be occupied is given by the composition profile in the intermixing region. For the layer with sharp interfaces the profile $\{\ldots 0,1,1, \ldots, 1,1,0, \ldots\}$ was taken [Fig. 5(a)], whereas in the case of nonsharp interfaces a linear profile, extending for $2 \mathrm{ML}$, was assumed 
(a)

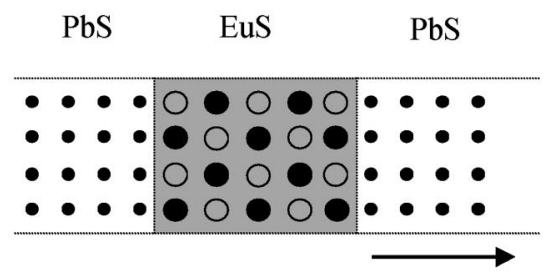

[100] (b)

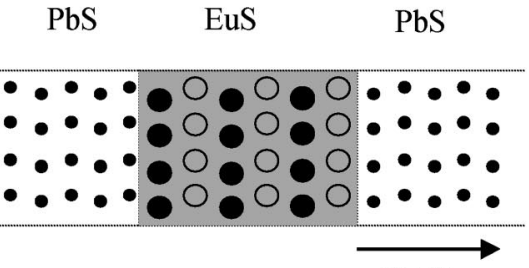

[111]
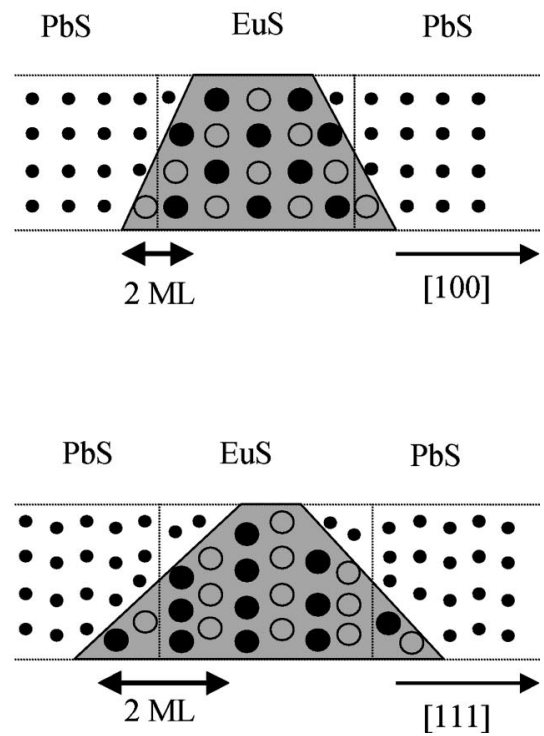

FIG. 5. Schematic presentation of the EuS-PbS structure grown in different crystallographic directions: [100] (KCl-based structure) and [111] $\left(\mathrm{BaF}_{2}\right.$-based structure). (a) shows the sharp interface, while (b) shows the case of an interface of 2-ML width. $\{\ldots, 0,1 / 3,2 / 3,1, \ldots, 1,2 / 3,1 / 3,0, \ldots\}$ [Fig. 5(b)]. This profile preserves the total number of spins. The final expression for $T_{C}^{(n)}$ reads

$$
T_{C}^{(n)}=T_{C}^{\mathrm{bulk}}(1-c / n),
$$

where $c$ is a numerical parameter. In Table II the parameters of $c$ and $T_{C}^{\text {bulk }}$, used for the calculations of $T_{C}^{(n)}$, were collected. The following values of exchange parameters ${ }^{15,22}$ were taken: $J_{\mathrm{NN}} / k_{B}=+0.22 \mathrm{~K}$ and $J_{\mathrm{NNN}} / k_{B}=-0.10 \mathrm{~K}$. In Fig. 4, we show the predictions of this "bond-loss" approach [Eq. (2)] with a sharp EuS-PbS interface (solid lines) as well as with a nonsharp interface (dashed line) both for $\mathrm{KCl}-$ and for $\mathrm{BaF}_{2}$-based series of structures. We note that this simple model provides a satisfactory description of the experimental data if one assumes an interface mixing of about 2 ML. This finding is in agreement with x-ray studies of the influence of temperature on interdiffusion process in $\mathrm{PbS}$-EuS structures, which suggest quite a low interdiffusion coefficient and a sharp EuS-PbS interface, of the order of 1-2 ML. ${ }^{37}$ We would like to stress that in the calculations presented in Fig. 4 the only adjustable parameter was the intermixing profile.

Since in our samples the ratio $d_{\mathrm{EuS}} / d_{\mathrm{PbS}}$ is not constant, and for pseudomorphically strained structures the strain is shared inversely proportional to thickness of the layers in the structure, ${ }^{28,41}$ we could expect the small effect of straininduced contribution to the $T_{C}(d)$ dependence, which we ignored in our derivation of Eq. (2). The compressing inplane stress of the EuS layer (due to the EuS-PbS lattice mismatch) could only intensify with decreasing EuS layer thickness and would result in an increase of $T_{C}$, i.e., the effect opposite to the prediction of "bond-loss" model and experimental observations. We can disregard this effect for EuS-PbS multilayers because it is not the EuS-PbS lattice mismatch but TEC-induced stress that is believed to be the dominant source of strain in these systems.

Our analysis of the thickness dependence of the Curie temperature $T_{C}\left(d_{\mathrm{EuS}}\right)$ of EuS-PbS multilayers presented above is based on the molecular field approach. This ap- proach turned out to be quite successful both for our layers and for other ferromagnetic ultrathin systems. ${ }^{45-47}$ However, the mean-field description of the ferromagnetic transition in low-dimensional magnetic systems with short-range interactions may serve only as the first approximation to the theoretical analysis of this transition. ${ }^{48-50}$ Below, we discuss the ferromagnetic transition in EuS-PbS multilayers within the framework of other concepts developed for the analysis of dimensional effects in low-dimensional magnetic systems.

The dependence of the critical temperature of the ferromagnetic phase transition $\left(T_{C}\right)$ on the thickness of the magnetic layers is frequently discussed in terms of the concept of finite-size scaling. ${ }^{48-50}$ This approach predicts, in particular, that the shift of the critical temperature $\left(T_{C}^{(n)}-T_{C}^{\text {bulk }}\right.$ ) is (for $n \rightarrow \infty)$ expected to vary as $n^{-\lambda}$, where $\lambda=1 / \nu_{3}$. Here $\nu_{3}$ $=2 / 3$ is the critical exponent for the magnetic correlation length of the 3D system. The characteristic dimensionality $D$ of a system is determined by the number of spatial dimensions in which the system has infinite extent. Accordingly, the layer of finite thickness $d(d \times \infty \times \infty)$ will exhibit twodimensional critical behavior as observed in purely $2 \mathrm{D}$ planar systems $(\infty \times \infty)$. In the light of the finite-size scaling theory, the crossover from $2 \mathrm{D}$ to $3 \mathrm{D}$ critical behavior of a system takes place when the temperature approaches the critical temperature $T_{C}^{(n)}$, and should occur when the magnetic correlation length $[\xi(T)]$ becomes of the order of the thickness of the layer $d$. Thus, sufficiently thin layers fulfill the criterion $d \approx \xi(T)$ at temperature $T$ well above the $T_{C}^{(n)}$, and then the nature of the phase transition will be analogous to 2D systems. Moreover, for two-dimensional (finite size) magnetic systems, the long-range order is expected to appear at lower temperatures than for the three-dimensional (bulk) systems.

As we mentioned before, EuS bulk crystals are isotropic 3D Heisenberg systems exhibiting a ferromagnetic phase transition. It is well known that the 2D Heisenberg system does not order at any finite temperature. ${ }^{45}$ However, it is expected that a 2D Heisenberg system with anisotropy will show a 2D Ising-like transition. ${ }^{49,50}$ Our FMR studies that 


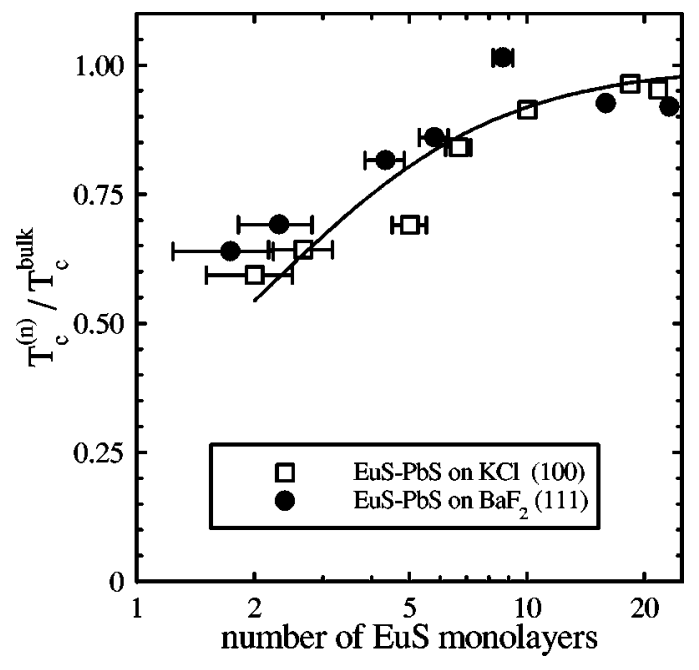

FIG. 6. The thickness dependence of the (normalized) ferromagnetic critical temperature $T_{C}^{(n)} / T_{C}^{\text {bulk }}$ for two series of EuS-PbS multilayers grown on the $\mathrm{KCl}$ (100) substrate (open squares) and on the $\mathrm{BaF}_{2}$ (111) substrate (full circles). The solid line is the result of calculations for ultrathin ferromagnetic Ising layers (Ref. 42) [Eq. (3)].

will be presented in Sec. V clearly show that in EuS-PbS multilayers the dominant source of magnetic anisotropy is the shape anisotropy resulting from dipole-dipole interactions, by which magnetization vector will stay in the plane of the EuS layer that corresponds to the effective $X Y$ spin dimensionality of the EuS thin layers. In this respect, EuS-PbS structures behave in a way well known for metallic ferromagnetic layers. ${ }^{7}$ One can expect that the weak in-plane anisotropy (see Sec. V) could cause the Ising-like effective spin dimensionality of EuS-PbS multilayers. ${ }^{4,48}$ Our attempt to verify experimentally this lattice and spin dimensionality crossover in EuS-PbS multilayers by studing the critical index of magnetic susceptibility turned out to be unsuccessful because of the considerable rounding-off of the sharp limiting phase transition.

We compared the obtained thickness dependence of $T_{C}^{(n)}$ for our EuS layers with the results of exact calculations performed for ferromagnetic Ising ultrathin layers containing $n$ monolayers. ${ }^{49,51}$ The calculations were done for $n$ $=3,4, \ldots, 10$ fcc lattice (mono)layers with free-surface boundary conditions. The extrapolation of high-temperature expansion series for zero-field magnetic susceptibility was used to estimate the critical temperatures. The dependence of the Curie temperature [given in terms of the parameter $\left.\mathrm{V}_{c}(n)=\tanh \left(J / k T_{C}^{(n)}\right)\right]$ on the thickness of the layer (given in terms of the number $n$ of monolayers) is given by ${ }^{47,51}$

$$
\mathrm{v}_{c}(n) \approx \mathrm{v}_{c}(\infty)+\frac{0.350}{(n+1 / 2)^{\lambda}},
$$

where $\lambda=1.56$. The temperature shift exponent $\lambda=1 / \nu_{3}$ $=1.56$ agrees well with the value $3 / 2$ expected from the $d$ $\approx \xi\left(T_{C}^{(n)}\right)$ criterion used in the finite scaling analysis of the Curie temperature shift $\left(T_{C}^{(n)}-T_{C}^{\text {bulk }}\right)$. In Fig. 6, our experimental data obtained for EuS-PbS layers are compared with the predictions of Eq. (3). It has to be noted that the calculations were performed for model Ising ferromagnets and do

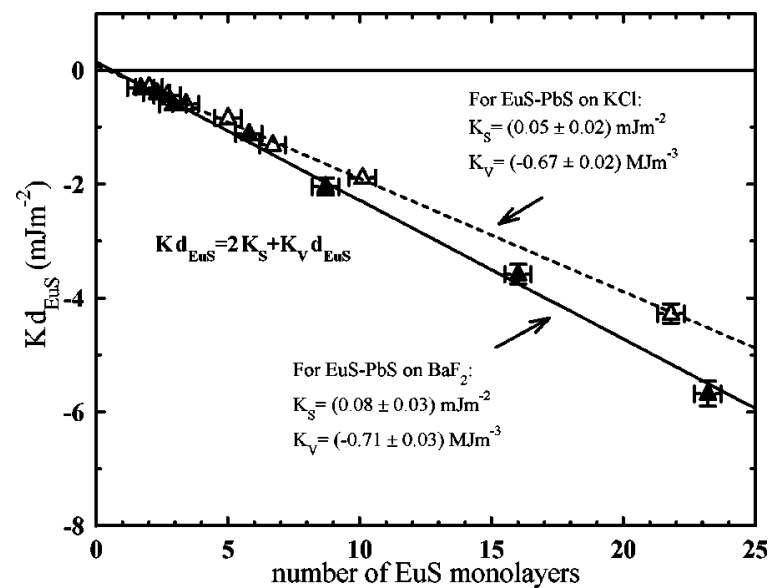

FIG. 7. The thickness dependence of the magnetic anisotropy per unit area for two series of EuS-PbS multilayers grown on the $\mathrm{KCl}$ (100) substrates (open triangles) and on the $\mathrm{BaF}_{2}$ (111) substrates (full triangles).

not take into account such factors as the interactions between $\mathrm{NNN}$, the intermixing of magnetic and nonmagnetic layers at the interface, and the nonzero magnetic field applied in the experiment. These factors might be relevant for a realistic description of the EuS-PbS system. In spite of its model character, Eq. (3) describes our data rather well over the whole measured range.

\section{MAGNETIC ANISOTROPY}

The EuS-PbS multilayers grown on $\mathrm{KCl}$ (100) and on $\mathrm{BaF}_{2}$ (111) substrates were also studied by ferromagnetic resonance (FMR). The primary aim of these measurements was to determine the dependence of the magnetic anisotropy on the thickness of the EuS layer. We have studied the temperature dependence of the position of the FMR line both in the configuration with magnetic field in the plane of the layer $\left(H_{\|}\right)$and in the configuration with magnetic field normal to the plane of the layer $\left(H_{\perp}\right)$. At temperatures $T \ll T_{C}$, we also examined the complete angle dependence of the resonance line position. The details of these measurements will be discussed elsewhere. The main conclusions relevant for this paper are as follows.

From the analysis of the temperature dependence of both the $H_{\|}$and $H_{\perp}$ we determined the ferromagnetic transition temperature $T_{C}$, which was found to follow the thickness dependence already discussed in Sec. IV. Since the temperature control in the FMR experiment is somewhat worse than in the SQUID or in the magnetic susceptibility measurements, and the FMR involves the application of a nonnegligible external magnetic field, the $T_{C}\left(d_{\text {EuS }}\right)$ dependence serves only as an additional confirmation of the other data shown in Fig. 4.

From the analysis of the angle dependence of the FMR line position, we determined the effective magnetization and magnetic anisotropy energy of EuS layers. The reduction of the effective magnetization of the thin layer from the value observed in bulk crystals is attributed to the magnetic surface anisotropy. We observe in Fig. 7 that the separation of the volume $\left(K_{V}\right)$ and surface $\left(K_{S}\right)$ anisotropy terms can be done by a common practice in the field ${ }^{7}$ using a $K d$ vs $d$ plot. The 
experimental data are well described by the relation $K\left(d_{\mathrm{EuS}}\right)=K_{V}+2 K_{S} / d_{\mathrm{EuS}}$, and for EuS-PbS structures on the $\mathrm{KCl}$ substrate the anisotropy coefficients are $K_{V}=$ $-0.67( \pm 0.02) \mathrm{MJ} \mathrm{m}^{-3}$ and $K_{S}=0.05( \pm 0.02) \mathrm{mJ} \mathrm{m}^{-2}$, while for the $\mathrm{BaF}_{2}$ case, $K_{V}=-0.71( \pm 0.03) \mathrm{MJ} \mathrm{m}^{-3}$ and $K_{S}=0.08( \pm 0.03) \mathrm{mJ} \mathrm{m}^{-2}$.

One can notice that for the EuS-PbS layers the surface term becomes dominant only for extremely thin layers of $\operatorname{EuS}\left(d_{\mathrm{EuS}} \leqslant 2 \AA\right)$. Since this thickness is below $1 \mathrm{ML}$, one may conclude that the EuS-PbS multilayers always magnetize in the plane of the structure and this process is driven by a dominant shape anisotropy of the EuS layers. The magnetic anisotropy contribution $K \propto 1 / d$ is likely to arise from the lowering of the symmetry of the magnetic layers at the EuS$\mathrm{PbS}$ interface. ${ }^{7,45}$ Since in our structures the dominant source of stress is expected to be the TEC-induced contribution, we do not expect the $1 / d$ contribution due to magnetoelastic effects. ${ }^{45}$ This mechanism is likely to contribute to EuS volume anisotropy constant and might partially explain small difference of volume contributions observed for layers grown on different substrates. The larger contribution of surface anisotropy observed for $\mathrm{BaF}_{2}$-based layers as compared to $\mathrm{KCl}$ based layers is in accordance with our suggestion (Sec. III) of lower symmetry of EuS layers at the EuS-PbS interface in $\mathrm{BaF}_{2}$ (111)-based structures as compared to $\mathrm{KCl}$ (100)-based structures.

We would also like to mention that the analysis of the width of the FMR line may serve as an indication about the quality of the layers. For our multilayer structures the peak to peak width of the $H_{\|}$resonance is $\Delta H_{\mathrm{pp}} \approx 300-500 \mathrm{G}$ for structures grown on the $\mathrm{KCl}(100)$ substrate and $\Delta H_{\mathrm{pp}}$ $\approx 400-600 \mathrm{G}$ for structures grown on the $\mathrm{BaF}_{2}$ (111) substrates. This result would indicate somewhat better (magnetic) perfection of $\mathrm{KCl}$-based structures. Comparing to other thin ferromagnetic layers such as $\mathrm{Co}-\mathrm{Cu}$ multilayers, ${ }^{44}$ the FMR linewidth observed in EuS-PbS structures is relatively large.

Additionally, one expects also a weak anisotropy in the plane of the layer due, in particular, to the single ion anisotropy of $\mathrm{Eu}^{2+}$ ions. This effect is well known in electron paramagnetic resonance studies of $\mathrm{Eu}^{2+}$ ions and it originates from the small influence of the electric field of ligand ions on the ground state of the $\mathrm{Eu}^{2+}$ ion in the crystal lattice. To obtain the information on the in-plane magnetic anisotropy we have studied the angle dependence of FMR line position with the magnetic field directed along different crys- tallographic directions in the plane of the layer. The in-plane angle dependence of FMR line was, however, barely resolved indicating the in-plane anisotropy field much smaller than width of the line, i.e., about 100-200 G.

\section{CONCLUSIONS}

We have studied the dependence of the magnetic properties of semiconducting ferromagnetic multilayers EuS-PbS/ $\mathrm{KCl}$ and $\mathrm{EuS}-\mathrm{PbS} / \mathrm{BaF}_{2}$ on the thickness of the EuS layer. The substantial reduction of the ferromagnetic Curie temperature $T_{C}$ is observed for structures with decreasing EuS layer thickness below $d_{\text {EuS }}<10$ ML. This effect can be qualitatively described taking into account the reduction of the number of magnetic neighbors for Eu ions located at (or close to) the interface between ferromagnetic EuS and diamagnetic $\mathrm{PbS}$. To reproduce the experimental $T_{C}\left(d_{\mathrm{EuS}}\right)$ dependence one has to assume an interface width of $2 \mathrm{ML}$. The thickness dependence of $T_{C}$ calculated exactly for ferromagnetic Ising ultrathin layers ${ }^{49,51}$ also describes our data rather well.

The energy of magnetic anisotropy $K_{\text {EuS }}$ was determined from the temperature and the angle dependence of the ferromagnetic resonance line. We found that the $K\left(d_{\mathrm{EuS}}\right)$ dependence can be described by the well-known expression $K$ $=K_{V}+2 K_{S} / d_{\text {EuS }}$ with the dominant role of the volume $K_{V}$ term (shape anisotropy). Our results show that the thickness of the EuS layer required for the perpendicular (to the layer) magnetization of the structure is about $2 \AA$, i.e., it is below 1 ML thickness.

We have also studied the role of stress present in our structures due to the difference between thermal expansion coefficients of the substrates and the structures. The semiquantitative analysis of this effect indicates that it might be responsible for a few Kelvin shift of the Curie temperature, and it is expected to operate differently for $\mathrm{KCl}(100)$ and for $\mathrm{BaF}_{2}$ (111) substrates. The effect of thermal stress on Curie temperature of EuS-PbS structures on $\mathrm{KCl}$ was experimentally demonstrated by magnetic susceptibility measurements of EuS layers on the substrate and the same layer without the substrate (removed by dissolving in water).

\section{ACKNOWLEDGMENT}

This work was supported in part by the Polish Committee for Scientific Research under Grant No. 2 P03B 10912.
${ }^{1}$ P. Grünberg, R. Schreiber, Y. Pang, M. B. Brodsky, and H. Sowers, Phys. Rev. Lett. 57, 2442 (1986).

${ }^{2}$ A. Fert, P. Grünberg, A. Barthélémy, F. Petroff, and W. Zinn, J. Magn. Magn. Mater. 140-144, 1 (1995), and references therein.

${ }^{3}$ Ultrathin Magnetic Structures, edited by J. A. C. Bland and B. Heinrich (Springer-Verlag, Berlin, 1994).

${ }^{4}$ Shi-Ming Zhou, Liagn-Yao Chen, Yi Su, You-Hua Qian, ZhiHong Jiang, and De-Fang Shen, J. Magn. Magn. Mater. 132, 219 (1994).

${ }^{5}$ P. Bruno, Phys. Rev. B 52, 411 (1995).

${ }^{6}$ T. M. Giebułtowicz, V. Nunez, G. Springholz, G. Bauer, J. Chen,
M. S. Dresselhaus, and J. K. Furdyna, J. Magn. Magn. Mater. 140-144, 635 (1995).

${ }^{7}$ W. J. M. de Jonge, P. J. H. Bloemen, and F. J. A. den Broeder, in Ultrathin Magnetic Structures I, edited by J. A. C. Bland and B. Heinrich (Springer-Verlag, Berlin, 1994), p. 65, and references therein.

${ }^{8}$ M. N. Baibich, J. M. Broto, A. Fert, F. Nguyen Van Dau, F. Petroff, P. Etienne, G. Creuzet, A. Friederich, and J. Chazelas, Phys. Rev. Lett. 61, 2472 (1988).

${ }^{9}$ G. Binasch, P. Grünberg, F. Saurenbach, and W. Zinn, Phys. Rev. B 39, 4828 (1989). 
${ }^{10}$ P. A. A. van der Heijden, P. J. H. Bloemen, J. M. Metselaar, R. M. Wolf, J. M. Gaines, J. T. W. M. van Eemeren, P. J. van der Zaag, and W. J. M. de Jonge, Phys. Rev. B 55, 11569 (1997); P. A. A. van der Heijden, C. H. W. Swüste, W. J. M. de Jonge, J. M. Gaines, J. T. W. M. van Eemeren, and K. M. Schep, Phys. Rev. Lett. 82, 1020 (1999).

${ }^{11}$ B. T. Matthias, R. M. Bozorth, and J. H. Van Vleck, Phys. Rev. Lett. 26, 1241 (1961).

${ }^{12}$ T. Kasuya, IBM J. Res. Dev. 14, 214 (1970); Crit. Rev. Solid State Sci. 3, 131 (1972).

${ }^{13}$ W. Zinn, J. Magn. Magn. Mater. 3, 23 (1976).

${ }^{14} \mathrm{P}$. Wachter, in Handbook on the Physics and Chemistry of the Rare Earths, edited by K. A. Gschneidner, Jr., and L. Eyring (North Holland, Amsterdam, 1979), Vol. 2, p. 507.

${ }^{15}$ A. Mauger and C. Godart, Phys. Rep. 141, 51 (1986).

${ }^{16}$ T. R. McGuire and M. W. Shafer, J. Appl. Phys. 35, 984 (1964).

${ }^{17}$ P. Wachter, Crit. Rev. Solid State Sci. 3, 189 (1972).

${ }^{18}$ G. Will, S. J. Pickart, H. A. Alperin, and R. Nathans, J. Phys. Chem. 24, 1679 (1963)

${ }^{19}$ N. F. Oliveira, Jr., S. Foner, Y. Shapira, and T. B. Reed, Phys. Rev. B 5, 2634 (1972).

${ }^{20}$ W. White, D. C. McCollum, and J. Callaway, Phys. Lett. 25A, 388 (1967)

${ }^{21}$ T. R. McGuire, B. E. Argyle, M. W. Shafer, and J. S. Smart, Appl. Phys. Lett. 1, 17 (1962).

${ }^{22}$ H. G. Bohn, W. Zinn, B. Dorner, and A. Kollmar, J. Appl. Phys. 52, 2228 (1981).

${ }^{23}$ L. Passell, O. W. Dietrich, and J. Als-Nielsen, Phys. Rev. B 14, 4897 (1976).

${ }^{24}$ B. Saftić, N. Rašula, W. Zinn, and J. Chevallier, J. Magn. Magn. Mater. 28, 305 (1982).

${ }^{25}$ S. Demokritov, U. Rücker, and P. Grünberg, J. Magn. Magn. Mater. 163, 21 (1996).

${ }^{26}$ H. Hori, M. Furusawa, R. Akimoto, M. Kobayashi, I. Kakeya, and K. Kindo, Physica B 216, 347 (1996).

${ }^{27}$ J. Blinowski and P. Kacman, Acta Phys. Pol. A 92, 719 (1997).

${ }^{28}$ J. Heremans and D. L. Partin, Phys. Rev. B 37, 6311 (1988).

${ }^{29}$ D. Kostyk, Y. Shapira, E. J. McNiff, Jr., T. Q. Vu, and A. Twardowski, Solid State Commun. 92, 473 (1994).

${ }^{30}$ L. Bergomi and J. J. Chen, Phys. Rev. B 56, 3281 (1997).

${ }^{31}$ Z. Wilamowski, R. Buczko, W. Jantsch, M. Ludwig, and G. Springholz, Acta Phys. Pol. A 90, 973 (1996).

${ }^{32}$ J. Wosnitza, H. V. Löhneysen, U. Walz, and W. Zinn, Z. Phys. B 77, 25 (1989).
${ }^{33}$ U. Rücker, S. Demokritov, R. R. Arons, and P. Grünberg, J. Magn. Magn. Mater. 156, 269 (1996).

${ }^{34}$ R. Dornhaus, G. Nimtz, and B. Schlicht, Narrow-gap Semiconductors (Springer-Verlag, Berlin, 1983).

${ }^{35}$ I. V. Kolesnikov and A. Yu. Sipatov, Fiz. Tekh. Poluprovodn. 23, 954 (1989) [Sov. Phys. Semicond. 23, 598 (1989)].

${ }^{36}$ I. V. Kolesnikov, V. A. Litvinov, A. Yu. Sipatov, A. I. Fedorenko, and A. E. Yunovich, Zh. Éksp. Teor. Fiz. 97 (7), 239 (1988) [Sov. Phys. JETP 67, 1431 (1988)].

${ }^{37}$ A. G. Fedorov, A. Yu. Sipatov, and E. V. Kaidalova (unpublished).

${ }^{38}$ A. Stachow-Wójcik, A. Twardowski, T. Story, W. Dobrowolski, E. Grodzicka, and A. Sipatov, Acta Phys. Pol. A 92, 985 (1997).

${ }^{39}$ L. Kowalczyk, J. Sadowski, R. R. Gałązka, A. Stachow-Wójcik, A. Yu. Sipatov, V. V. Volobuev, V. A. Smirnov, and V. K. Dugaev, Acta Phys. Pol. A 94, 397 (1998).

${ }^{40}$ I. N. Goncharenko and I. Mirebeau, Phys. Rev. Lett. 80, 1082 (1998).

${ }^{41}$ Strained Layers Superlattices, edited by R. K. Willarson and A. C. Beer, Semiconductors and Semimetals, Vols. 32 and 33 (Academic, San Diego, California, 1990).

${ }^{42}$ G. Bauer, Semicond. Sci. Technol. 5, 122 (1990).

${ }^{43}$ M. E. Fisher, in Critical Phenomena, Proceedings of the International School of Physics "Enrico Fermi," Course LI, Varenna, Italy, 1970, edited by M. S. Green (Academic, New York, 1971).

${ }^{44}$ H. A. M. de Gronckel, K. Kopinga, W. J. M. de Jonge, P. Panissod, J. P. Schille, and F. J. A. den Broeder, Phys. Rev. B 44, 9100 (1991).

${ }^{45}$ C. Chappart and P. Bruno, J. Appl. Phys. 64, 5736 (1988).

${ }^{46}$ W. Durr, M. Taborelli, O. Paul, R. Germar, W. Gudat, D. Pescia, and M. Landolt, Phys. Rev. Lett. 62, 206 (1989).

${ }^{47}$ C. M. Schneider, P. Bressler, P. Schuster, J. Kirschner, J. J. de Miguel, and R. Miranda, Phys. Rev. Lett. 64, 1059 (1990).

${ }^{48}$ See, e.g., M. E. Fisher and M. N. Barber, Phys. Rev. Lett. 28, 1516 (1972); M. N. Barber, Phys. Rev. B 8, 407 (1973); K. Binder and P. C. Hohenberg, ibid. 6, 3461 (1972); P. G. Watson, in Phase Transitions and Critical Phenomena, edited by C. Domb and M. S. Green (Academic, London, 1972), Vol. 2.

${ }^{49}$ T. W. Capehart and M. E. Fisher, Phys. Rev. B 13, 5021 (1976).

${ }^{50}$ N. D. Mermin and H. Wagner, Phys. Rev. Lett. 17, 1133 (1966).

${ }^{51}$ See for review: Magnetic Properties of Layered Transition Metal Compounds, edited by L. J. de Jongh (Kluwer Academic, The Netherlands, 1990), pp. 1-51. 